\section{How to make a CRISPR cow} to insert large DNA fragments their knowledge of bovine
Genome editing offers an opportunity to introduce use genetic traits into livestock

breeding programmes. into livestock embryos using Alison Van Eenennaam. and Joseph Owen of the University of California, Davis, employed embryogenesis and DNA repair pathways, and the help of a green fluorescent reporter gene, to generate a CRISPR calt named Cosmo who carrie SRY copies of th

or centuries, breeders have selected animals displaying desirable qual generation. When a breeder chooses animal based on a visible characteristic - for example, coat colour - they are actually selecting between the naturally occurring variations in DNA sequences sponsible for differences between individuals.

Using conventional breeding, achieving the desired changes can take decades. This is especially true of large livestock species like cattle, which have long generation intervals. Advances in gen editing offer an opportunity to bring In this process, specific DNA sequence variations can be inserted directly into livestock genomes.

One common method used to edit genom is the CRISPR/Cas9 system

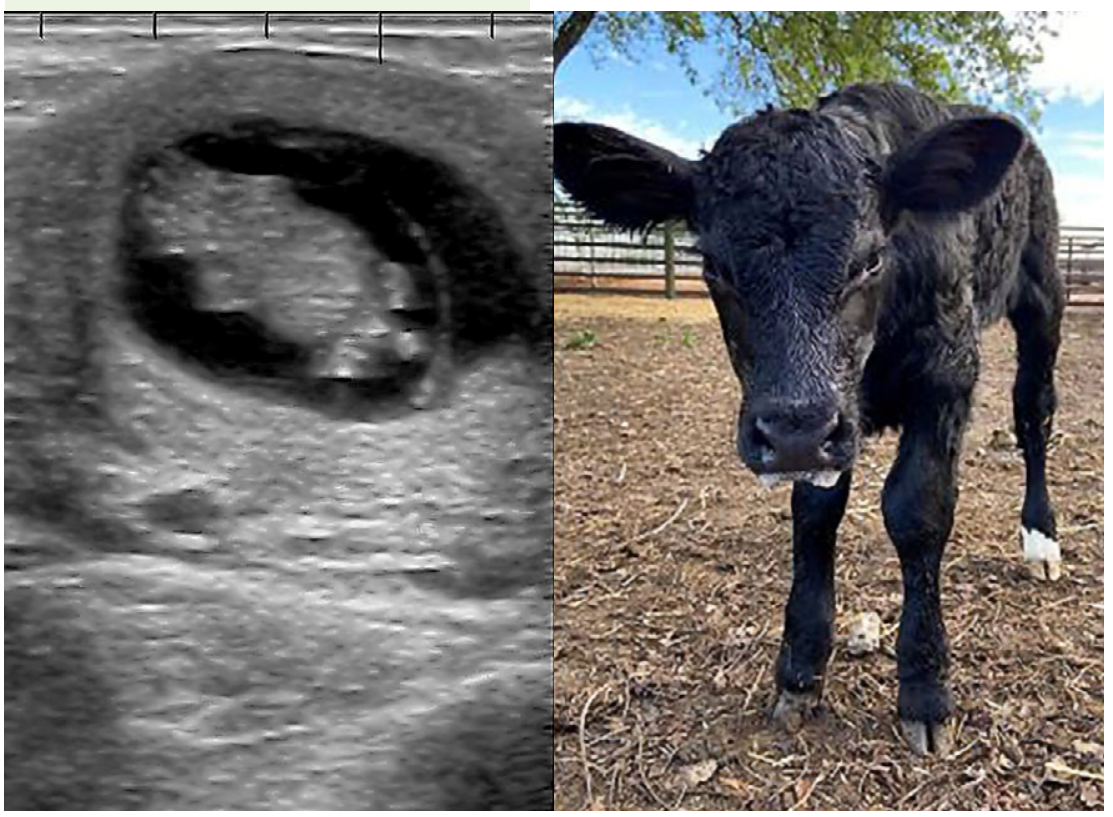

Cosmo: a targeted knock-in calf developed using the CRISPR-Cas9 $9 y$ stem in bovine zygotes. about useful changes far more quickly.
This system has two components. The Cas9 protein, which cuts DNA, and a guide molecule that directs Cas 9 where to cut. Bound together, they form a complex that cuts DNA at the target site. The resulling break, called a DNA double cell's break (DSB), is then fixed by the

Cells use different natural mechanisms The nonhomologous end joining (NHEJ) pathway and the homology-directed repair (HDR) pathway are two possible repair routes. NHEJ typically leads to short insertion deletion events (where a tiny fragment of DNA is inserted into or deleted site. Alternatively, HDR can be used to introduce useful DNA sequences from a donor template. The HDR donor repair template typically harbours the flanked on either side by homology arms. These arms mirror the sequence
of the DNA on either side of the CRISPR target site. So far, it has proven to be very difficult to insert whole genes pathway in embryos.

Recently, a team led by Dr Alison Van Eenennaam and PhD student Joseph Owen at the University of California, Davis, used the CRISPR-Cas9 system in early bovine embryos to produce a calf carrying targeted insertions of the SRY gene. The bull calt, named Cosmo, was bom in April 2020 during the COVID-19 pandemic. Cosmo is unique in that he pros carres copies of a green fluorescent a marker to idurify ence was used as surrogate cows, and one subsequently gave birth to Cosmo. from the genome) near the cutting DNA fragment or gene to be inserted the targeted SRY gembryos carrying embryos were then transert. Those

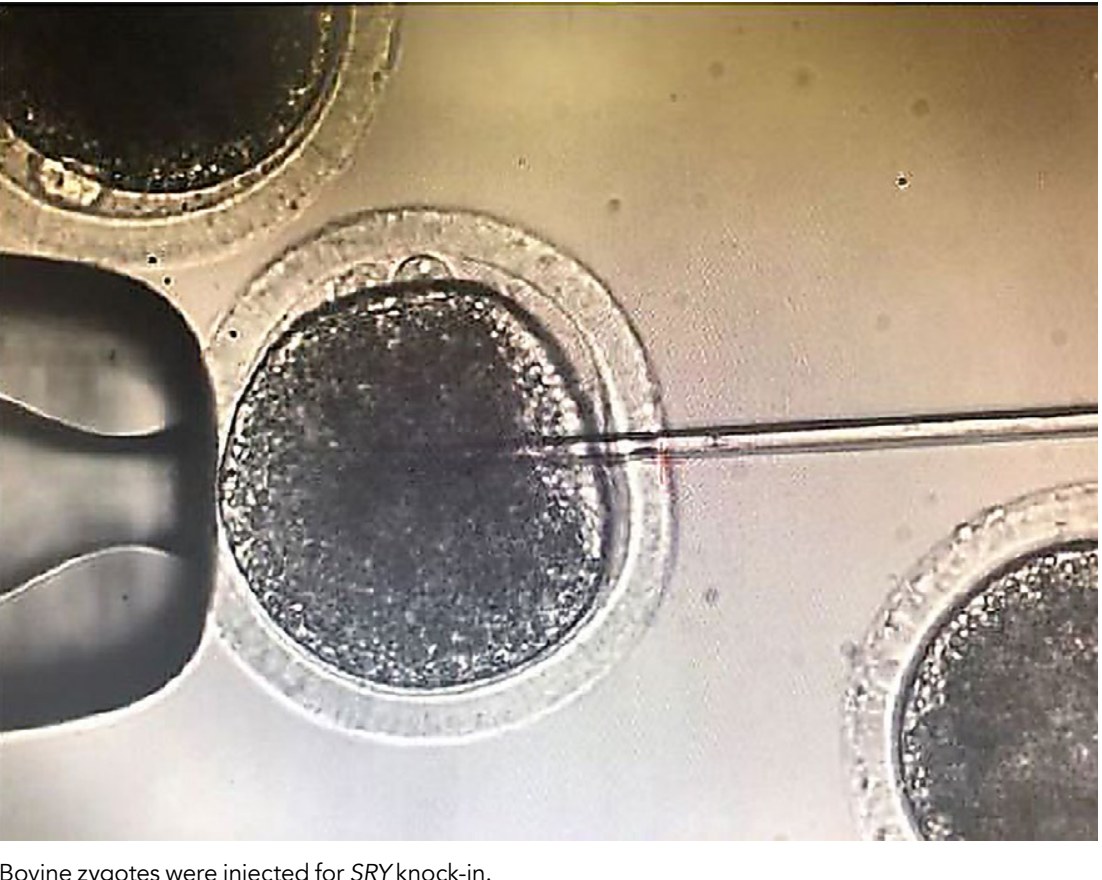

To avoid the insertion of the new gene being thwarted by the cell's DNA repair system, the team employed a homology-mediated end joining (HMEJ) strategy。

\section{WHY THIS GENE?}

The SRY gene carries the instructions for a protein called the sex-determining region $Y$, which is involved in male sexual development. Specifically, this protein acts as a transcription factor, which means it attaches to specific ectivity of particur tarts processes that cause a foetus to der a mas (testes) and prevent the development of female reproductive structures (uterus and fallopian tubes). Inheriting the SRY gene causes an embryo to develop as a male. Bulls that could produce all-male offspring would be desirable for beef farmers; in the same way, bulls that sire all-female offspring would be desirable for dairy farmers.

\section{DESIGNING THE GUIDE}

As a first step in the insertion of the SRY gene into the embryo, the team developed a guide RNA. This guide RNA (single-stranded nucleic acid, directed the Cas? proten to cut a
TIMING IS EVERYTHING

One of the aims of this research was to fertilisation to avoid a condition called genetic mosaicism. Mosaisism occurs when two or more groups of cells with different genotypes are present within an individual that has developed from a single fertilised egg. This usually happens when editing takes place during or after the embryo begins replicating its own DNA just before the first cell division. To help avoid mosaicism in this research, the guide, were introduced into bovine embryos just six hours after insemination, and One of the challenges with this timing for researchers hoping to insert a gene predominant DNA repair pathway. In the pearly stages of embryo developm. In the repair tends to be via the NHEJ pathway whereas HDR editing efficiency is very low in early embryos.

To avoid the insertion of the new gene being thwarted by the cell's DNA homology-mediated end joining (HMEJ) strategy. In this process, HMEJ donor templates are designed so that the homology arms are flanked on both sides by the CRISPR target sequence. This HMEJ-based approach has proven to be effective in early stage 1-cell much greater than that of alternative SRY designed a HMEJ donor template, with either side of the homology arms.

However, insertion using this method is still not $100 \%$ efficient. In initial donor and Cas9 protein editing regents before the beginning of DNA synthesis.

\section{AN INSERTION STRATEGY} repair system, the team employed a embryos, with a gene insertion efficien the H11 CRISPR target sequence on

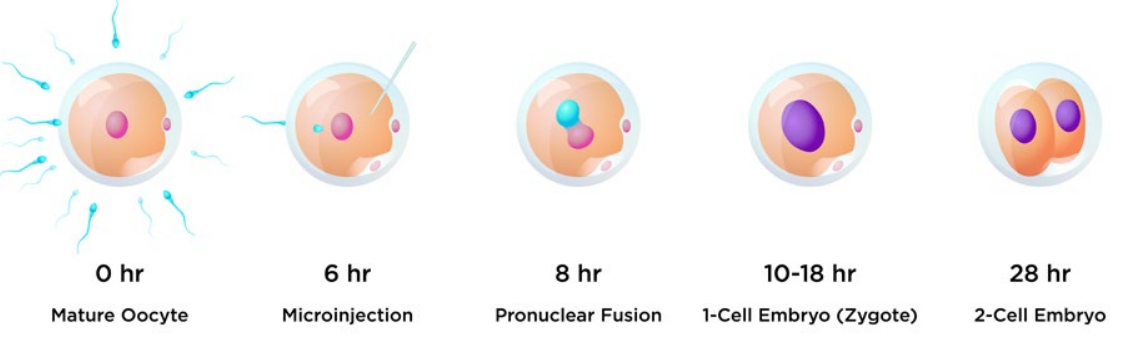

The timing of the microinjection of the editing regents is key: Lower rates of mosaicism were observed
when injecting into embryos 6 hours after fertilisation, prior to DNA replication and the first cell division. 


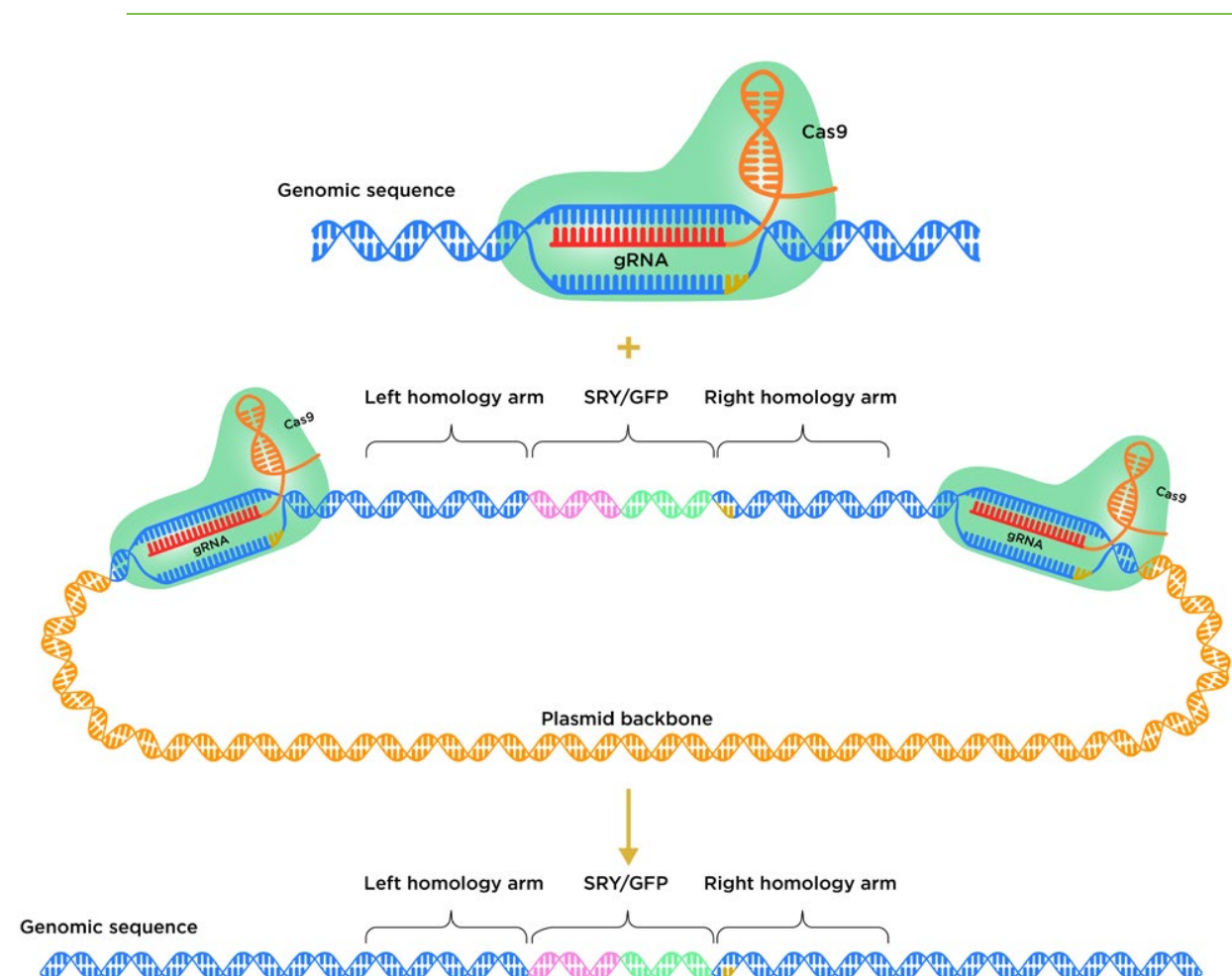

Zygotes iniected with the HMEJ-based donor plasmid and the CRISPR-Cas9 genome editing reagents
showed high rates of integration of SRY at target site.

The world's first knock-in bull by CRISPRmediated HMEJ in early embryos

weighed 110 pounds $(50 \mathrm{~kg})$ at birth.

experiments, only around $40 \%$ of the bovine embryos were found to have the $S R Y$ insertion. Bovine embryo transfers, and the subsequent nine-month pregnancy, are expensive. This mean the team needed to confirm whether the gene insertion had been successful before he com asing encio cells are removed from the embry and then checked for the presence of the inserted SRY gene. Unfortunately, this embryo, especially if the embryos are then frozen for transfer at a later date.

The team opted for a different screening approach: a "reporter" gene. A reporter gene carries an easily identifiable characteristic - fluorescence, for example - which allows for noninvasive screening of embryos. In this experiment, the jellyfish green fluorescent protein (GFP), was included alongside SRY, nestled between the homology arms on the HMEJ do caused any embryo with an SRY insert to glow green when exposed to light in the blue to ultraviolet range. Embryos were screened, and in June 2019, nine 7-day embryos that fluoresced green under UV light were transfered to surrogate cows. A moth later one of those cows,

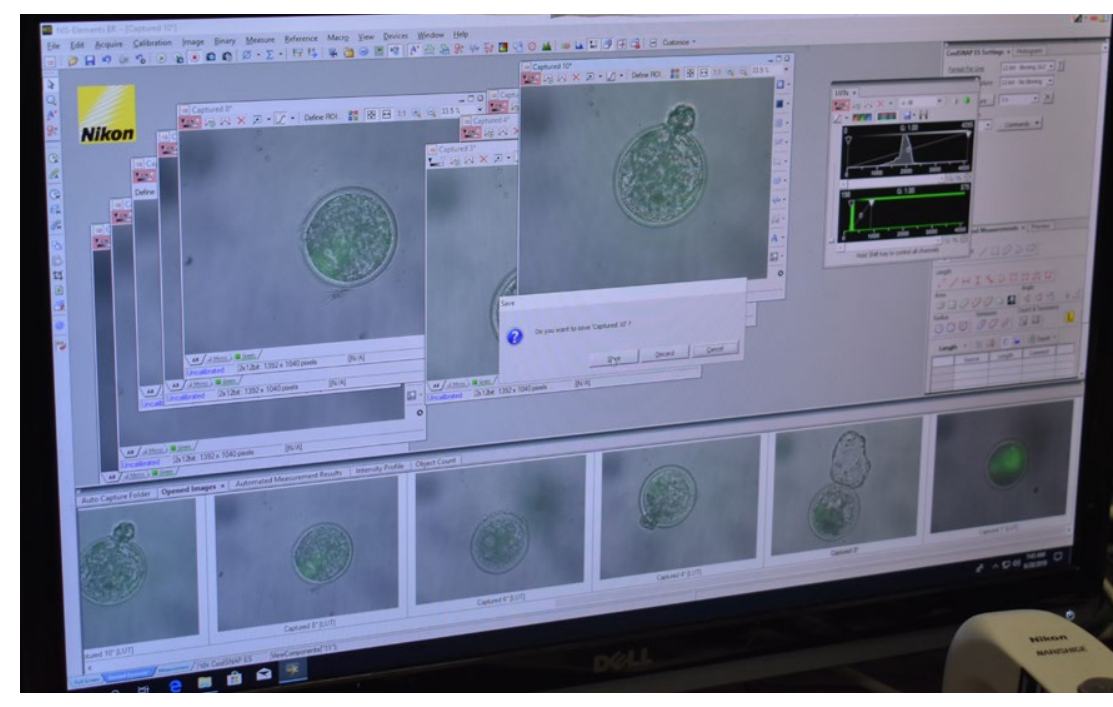

ultrasound scan revealed she was carrying a male bull calf.

COSMO'S ARRIVAL

Nine months later, during the

COVID-19 pandemic, Cosmo made bull by CRISPR-mediated HMEJ in early birth. The team were able to carry out detailed genetic analyses of the calf. They found that Cosmo carries several copies of the GFP:SRY gene on one of the Chromosome 17 pair, and a small insertion on the other. This suggests that, when Cosmo was a newly-formed embryo, the DSB introduced by Cas 9 at the H11 locus on one Chromosome 7 was repaired by NHEJ resulting in a small 26 base pair insertion, and the donor template to produce multiple GP nd SRY gene insertions, and a copy of the donor plasmid backbone.

The team hope that, one day in the future, Cosmo will become a father.

Cosmo's future offspring should reveal something interesting: will inheriting in bovine XX embryos (which would normally be female) developing into male calves? And, of course, Cosmo still carries several copies of the green

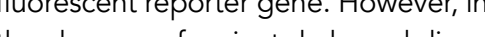
the absence of a giant darkened disco lounge where Cosmo could be exposed to light in the blue to ultraviolet range, the reschers wilprobably neverbe

screening of embryos. embryos weighed 110 pounds $(50 \mathrm{~kg})$ at several copies of the SRY gene result

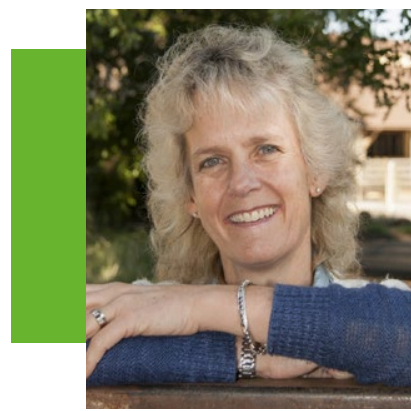

Behind the Research

\section{Dr Alison L. Van Eenennaam}

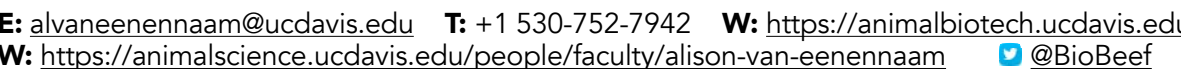
W: https: //animalscience.ucdavis.edu/pect
W: http://biobeef.faculty.ucdavis.edu

Research Objectives

DrVan Eenennaam's Animal Genomics and Biotechnology Program provides research and outreach materials on the

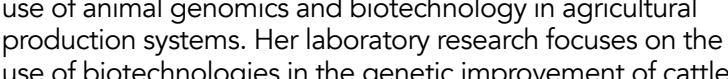

Detail

Department of Animal Science

2113 Meyer Hall

University of California
One Shields Avenue

Davis,

Bio

PAlison Van Eenennaam earned a Bachelor of Agricultural Science (Hons) from the University of Melbourne in Australi from University of California, Davis. For the past 18 years, she has been a Cooperative Extension Specialist in the field of Animal Science at UC Davis. Her current research focuses genome editing applications in livestock species.

Funding

This work was supported in part by grants from Biotechnology Risk Assessment Grant Program competitive grant no. 2015UC Davis Academic Federation Innovative Development Awards Grant Program; the Russell L. Rustici Rangeland and Cattle Research Endowment of the College of Agricultural and Environmental Sciences; and the California Agricultura

Collaborators

ment of Animal Science, UC

- Dr James D. Murray, Department of Animal Science and of Veterinary Medicine University of California, Davis CA - Dr Brett R. McNabb, Department of Population Health and Reproduction, School of Veterinary Medicine, University of

- Dr Tamer A. Manasour, Department of Clinical Pathology, Egypt E. Young, Center for Equine Health, School of - Sadie L. Hennig, Graduate Student, Department of Animal Science, UC Davis, CA Science, UC Davis, CA

UCDAVIS ANIMAL SCIENCE

\section{References}

Owen, J.R., et al. 2019. Improved rate of targeted gene knockin of in-vitro fertilized bovine embryos. Proc Assoc Advmt An
Breed Genet. 23:-7-10 http://www.aaabg. org/aaabghome/ Breed Genet. 23:7-10. http:///www.aa
AAABG23papers/2Owen23007.pdf

Hennig, S.L., et al. 2020. Evaluation of mosaicism and offtarget mutations in CRISPR-mediated genome edited bovine embryos. bioRxiv 2020.2006.2004.

Owen, J.R., et al. 2020. Harnessing endogenous repair mechanisms for targeted gene knock-in or bovine embryos.
Sci Rep. 2020. http://www.nature.com/articles/s41598-020$72902-x$

Owen, J.R., et al. 2020. PSX-32 - Late-Breaking Abstract: genome editing. $J$ Anim SCi. 98, Issue Supplement. In pes

\section{Personal Response}

This is a fascinating story. What are the next steps for your research?

III Fluorescent in situ hybridisation of chromosomal spread will be used to confirm the chromosomal location and zygosity of the Krock-in insertions. HII-C, Illumina short read and PacBio haplotype phase of Chromosome 17 and evaluate SNPs and alterations in the bull's genome. We also have a couple more cows that are pregnant with calves for a project where genome This project highlights some of the complexities of working with large livestock species. Future analysis of the XX offspring
inheriting the SPY gene on Chromosome 17 from this knock bull will revea whether inheritonce of the bovine SPY gene is sufficient to trigger the male developmental pathway in cattle. The $2-3$ year generation interval of cattle means it will be years We are working on methods to try to improve the efficiency than microinjection, to introduce the editing reagents into bovine embryos. We hope this will increase the efficiency of the editing process, and improve the survivability of edited embryos.

Another complication is the current regulatory approach genomic alterations introduced through gene editing in animals are regulated as veterinary drugs. This means the must go through a multigenerational safety and efficacy evaluation prior to entering commerce, which can become prohibitively expensive for small companies and academic
researchers working with large livestock species. 